\title{
Comparison of electronic structure and template function of single-layer graphene and a hexagonal boron nitride nanomesh on $\mathrm{Ru}(0001)$
}

\author{
Thomas Brugger, ${ }^{1}$ Sebastian Günther, ${ }^{2}$ Bin Wang, ${ }^{3}$ J. Hugo Dil, ${ }^{1,4}$ Marie-Laure Bocquet, ${ }^{3,2}$ Jürg Osterwalder, ${ }^{1}$ \\ Joost Wintterlin, ${ }^{2}$ and Thomas Greber ${ }^{1, *}$ \\ ${ }^{1}$ Physik-Institut, Universität Zürich, Winterthurerstrasse 190, CH-8057 Zürich, Switzerland \\ ${ }^{2}$ Department Chemie, Ludwig-Maximilian Universität, Butenandtstrasse 5-13, D-81377 München, Germany \\ ${ }^{3}$ Laboratoire de Chimie, Université de Lyon, Ecole Normale Supérieure de Lyon, CNRS, F-69007 Lyon, France \\ ${ }^{4}$ Swiss Light Source, Paul Scherrer Institute, CH-5232 Villigen, Switzerland
}

(Received 10 November 2008; published 12 January 2009)

\begin{abstract}
The structure of a single-layer graphene on $\mathrm{Ru}(0001)$ is compared with that of a single-layer hexagonal boron nitride nanomesh on $\mathrm{Ru}(0001)$. Both are corrugated $s p^{2}$ hybridized networks and display a $\pi$ band gap at the $\overline{\mathrm{K}}$ point of their $1 \times 1$ Brillouin zone. In contrast to $h$-BN/Ru(0001), $g / \mathrm{Ru}(0001)$ has a distinct Fermi surface. Together with the band structure measurements this indicates that $0.1 e$ per $1 \times 1$ unit cell are transferred from the $\mathrm{Ru}$ substrate to the graphene. Photoemission from adsorbed xenon on $g / \mathrm{Ru}(0001)$ identifies two Xe $5 p_{1 / 2}$ lines, separated by $240 \mathrm{meV}$, which reveals a corrugated electrostatic potential energy surface like on $h$-BN/Rh(111). These two Xe species are related to the topography of the template and have different desorption energies.
\end{abstract}

DOI: 10.1103/PhysRevB.79.045407

PACS number(s): 73.20.-r, 73.30.+y, 73.61.Wp, 79.60.Dp

\section{INTRODUCTION}

A single layer of an adsorbate strongly influences the physical and chemical properties of a surface. Sticking and bonding of atoms and molecules may change by orders of magnitude as well as the transport properties across and parallel to the interface. For developments in nanotechnology it is particularly useful to have single-layer systems which are inert, remain clean at ambient conditions, and are stable up to high temperatures. In this field $s p^{2}$ hybridized graphene $(g)$ and hexagonal boron nitride $(h-\mathrm{BN})$ are outstanding examples. ${ }^{1,2}$ On ruthenium both form perfect single layers, where the lattice mismatch between the substrate and the adsorbate causes two-dimensional regular superstructures with a lattice constant of about $3 \mathrm{~nm} \cdot{ }^{3-6}$ A layer of hexagonal boron nitride on $\mathrm{Rh}(111)$ (Ref. 2) and $\mathrm{Ru}(0001)$ (Ref. 6) which was coined $h$-BN nanomesh is now considered to be a corrugated single-layer dielectric. ${ }^{7}$ In the case of the $h$-BN/ $\mathrm{Rh}(111)$ nanomesh, which has an atomic and electronic structure similar to $h-\mathrm{BN} / \mathrm{Ru}(0001)$, single molecules separated by $3 \mathrm{~nm}$ are observed after adsorption at room temperature. ${ }^{7}$ This peculiar functionality as a template for trapping molecules might also be valid for $g / \mathrm{Ru}(0001)$, although experimental demonstrations on this issue are missing so far.

The purpose of this paper is to establish a comparison between $g / \mathrm{Ru}(0001)$ and $h-\mathrm{BN} / \mathrm{Ru}(0001)$ in view of the electronic structure and functionality as a template. For this purpose, photoemission and density functional theory (DFT) are used. It is shown that $g / \mathrm{Ru}(0001)$ is a metal with a sizable Fermi surface while $h-\mathrm{BN} / \mathrm{Ru}(0001)$ is not. The exploration of the electrostatic potential energy landscape by photoemission of adsorbed Xe also indicates a modulation of the local work function for $g / \mathrm{Ru}(0001)$, analog to the $h$-BN/ $\mathrm{Rh}(111)$ nanomesh ${ }^{8}$ and a concomitant template function. However, the different topography of the two systems also suggests complementary functionality.
The lattice mismatch $\mathfrak{M}$ of the laterally rigid $s p^{2}$ networks graphene and hexagonal boron nitride on different transition metals leads to superstructures with lattice constants in the $s p^{2}$ layer of $a_{s p^{2}} / \mathfrak{M}$, where $a_{s p^{2}}$ is the $1 \times 1$ lattice constant of graphene and $h$-BN, of about $0.25 \mathrm{~nm} .{ }^{9}$ Together with the coordination dependent bond strength of the adsorbate atoms to the transition metal substrate atoms, this leads to distinct atomic structures. ${ }^{2-6,10-16}$ For graphene on $\mathrm{Ru}(0001) 25 \times 25$ graphene unit cells coincide with 23 $\times 23 \mathrm{Ru}$ units. ${ }^{17}$ This large unit cell splits into four subunits of $12.5 \times 12.5 \mathrm{~g}$ on $11.5 \times 11.5 \mathrm{Ru}$ cells. Each subunit corresponds to one wavelength of the corrugation of the graphene overlayer. For $h$-BN/Rh(111) $13 \times 13 \mathrm{BN}$ units coincide with $12 \times 12 \mathrm{Rh}$ units. ${ }^{2,18}$ The accompanying variation in the local coordination of the substrate and the adsorbate atoms divides the unit cells into regions with different lateral coordination. The notation $(\mathrm{B}, \mathrm{N}) \sim($ top, hcp) refers to the local configuration, where a B atom sits on top of the substrate atom in the first layer and a $\mathrm{N}$ atom sits on top of the hexagonal-close-packed (hcp) site, which is on top of the substrate atom in the second layer. Three regions can be distinguished with atoms in (fcc,top), (top,hcp), and (hcp,fcc) configurations (see Fig. 1). Of course, because of the mismatch these local coordinations are only approximate and the transitions between them are not sharp. Whereas $h$-BN has a base with two different atoms in the unit cell, the base of graphene consists of two identical carbon atoms $\mathrm{C}_{\mathrm{A}}$ and $\mathrm{C}_{\mathrm{B}}$ which become distinguishable by the local coordination to the substrate. In $g / \mathrm{Ru}$ the local (fcc,top) and (top,hcp) coordinations lead to close contact between the $\left(\mathrm{C}_{\mathrm{A}}, \mathrm{C}_{\mathrm{B}}\right)$ atoms and the substrate ${ }^{19}$ while $(\mathrm{B}, \mathrm{N})$ is strongly interacting only in the (fcc,top) coordination. ${ }^{20}$ As a result, twice as many atoms are bound in strongly interacting regions in $g / \mathrm{Ru}$ when compared to $h$-BN/Ru. In the following we call the strongly bound or low region of $g / \mathrm{Ru}(0001)$ valley $(V)$ and the weakly bound or high region with the $\left(\mathrm{C}_{\mathrm{A}}, \mathrm{C}_{\mathrm{B}}\right)$ atoms on (hcp,fcc) sites mounds $(M)$. The fact that (top,hcp) 

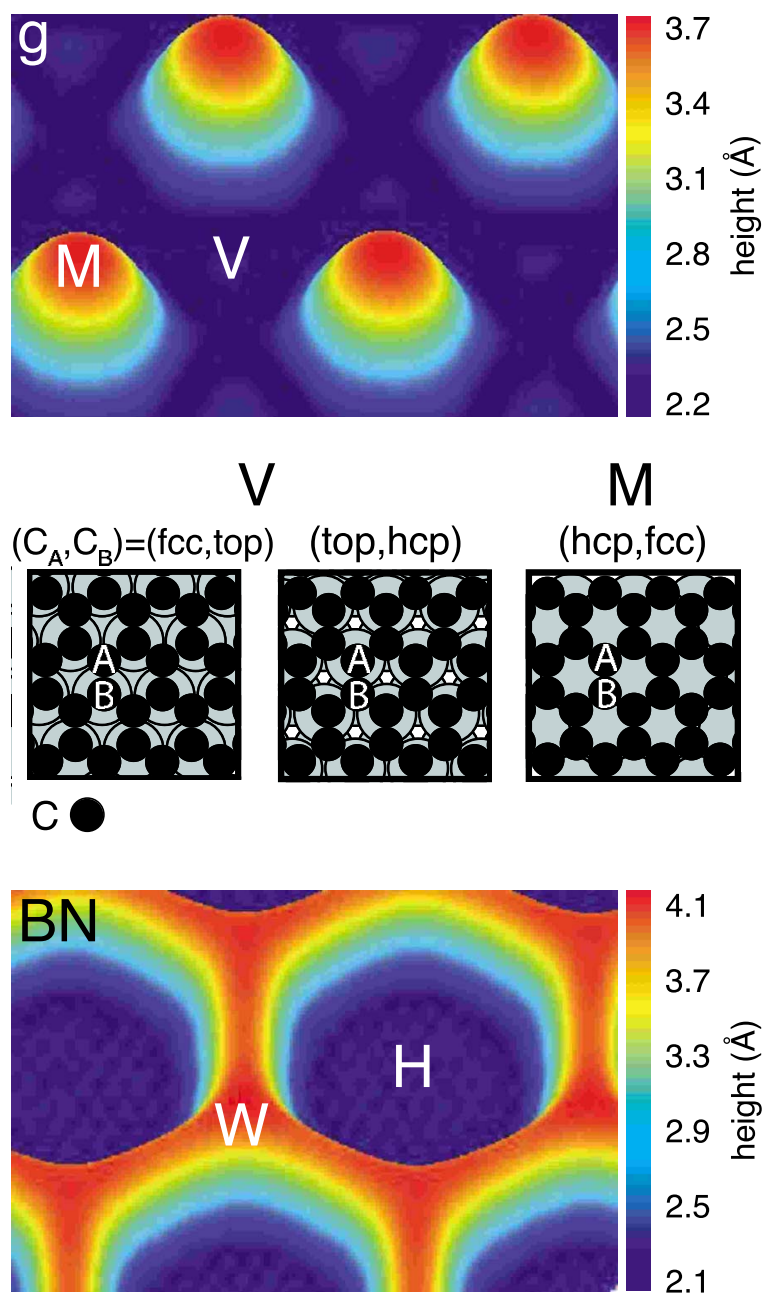

4.1

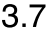

$3.3 \stackrel{\text { 爻 }}{2.9}$

2.5

2.1



FIG. 1. (Color online) Views of the height modulated graphene $(g)$ and $h$-BN nanomesh $(\mathrm{BN})$ on $\mathrm{Ru}(0001)$, as obtained from a DFT calculation for both systems. $M$ and $V$ denote mounds and valleys of the graphene, $H$ and $W$ holes and wires of the $h$-BN nanomesh. The six ball model panels illustrate the three different regions [(fcc,top), (top,hcp), and (hcp,fcc)] which can be distinguished in both systems (see text).

leads to strong bonding for graphene but weak bonding for $h$-BN gives rise to an inverted topography of the two layers: a connected network of strongly bound regions for graphene (valley) and a connected network of weakly bound regions for $h$-BN (wires).

The height in Fig. 1 corresponds to the theoretical results. It is the distance of the overlayer atoms from the average position of the first $\mathrm{Ru}$ layer. The value for the $h$-BN na- nomesh on $\mathrm{Ru}(0001)$ is larger than the presently accepted experimental value of $\approx 0.5 \AA$ (Ref. 6) and the theoretical value of $0.55 \AA$ for the $h$-BN nanomesh on $\operatorname{Rh}(111) .{ }^{21}$ For the case of $g / \mathrm{Ru}$ scanning tunneling microscopy (STM) indicates bias dependent mound heights of 0.7-1.1 $\AA$ (Ref. 3) and $0.2-1.0 \AA .5,22,23$ These quantitatively conflicting results indicate how difficult the complete theoretical description of the $s p^{2}$ layers with different bonding regimes is. It has, e.g., to be expected that van der Waals bonding, which is important for the mounds of $g / \mathrm{Ru}$ and the wires of $h-\mathrm{BN} / \mathrm{Ru}$, is less well described by DFT than the strongly bound regions. We emphasize that theory predicts the correct shape of the $s p^{2}$ layer corrugations while the absolute values of the heights that are reflected in the band splittings and the electrostatic potential corrugation are too large if compared with the experiment. The recent structure determination of $g / \mathrm{Ru}(0001)$ (Ref. 17) relied on the theoretical results of the graphene corrugation ${ }^{19}$ but demonstrated a substantial $\mathrm{Ru}$ substrate reconstruction. An unambiguous experimental $s p^{2}$ layer corrugation determination by structural methods will involve low-energy electron diffraction (LEED), atomic force microscopy, or He diffraction.

\section{EXPERIMENT}

The angle-resolved photoemission experiments were performed in a modified VG ESCALAB 220 (Ref. 24) using monochromatized $\mathrm{He} \mathrm{II}_{\alpha}$ radiation with a photon energy of $40.8 \mathrm{eV}$. The xenon experiments were accomplished in the COPHEE endstation ${ }^{25}$ at the Surface and Interface Spectroscopy beamline at the Swiss Light Source with monochromatized $\mathrm{He}_{\alpha}$ radiation of $21.2 \mathrm{eV}$ photon energy. A singlelayer graphene has been grown by thermal decomposition of $30 \mathrm{~L}\left(1 \mathrm{~L}=10^{-6}\right.$ Torr s) ethene $\left(\mathrm{C}_{2} \mathrm{H}_{4}\right)$ on the $1100 \mathrm{~K}$ hot $\mathrm{Ru}(0001)$ surface which had been cleaned by repeated $\mathrm{Ar}^{+}$ sputtering and annealing cycles. The $h$-BN nanomesh has been formed in a similar manner by decomposing $40 \mathrm{~L}$ of borazine $(\mathrm{HBNH})_{3}$ on the $1030 \mathrm{~K}$ hot substrate. The formation of the superstructures was confirmed by LEED and STM.

\section{THEORY}

$A b$ initio calculations were performed with the VASP package based on DFT, which implements projector augmented wave (PAW) pseudopotentials ${ }^{26}$ and the Perdew-BurkeErnzerhof (PBE) exchange-correlation functional in the generalized gradient approximation (GGA). ${ }^{27}$ The structural calculations of $\mathrm{g} / \mathrm{Ru}$ were performed with a $12 \times 12 \mathrm{~g}$ on $11 \times 11 \mathrm{Ru}$ supercell. It was tested that the results for a $13 \times 13 \mathrm{~g}$ on $12 \times 12 \mathrm{Ru}$ supercell were very similar so that the actual $12.5 \times 12.5 \mathrm{~g}$ on $11.5 \times 11.5 \mathrm{Ru}$ supercell also must be well described. For $h$-BN/Ru a $13 \times 13 \mathrm{BN}$ on 12 $\times 12 \mathrm{Ru}$ supercell was used. One layer of $g(\mathrm{BN})$ on a three layer $\mathrm{Ru}$ slab with the $g(\mathrm{BN})$ layer and the first $\mathrm{Ru}$ layer relaxed was investigated. The effective vacuum region was larger than $7.5 \AA$. For the band structure calculations epitaxial $1 \times 1 \mathrm{~g}$ or $\mathrm{BN} / \mathrm{Ru}$ structures were investigated with the lattice constant of graphene kept fixed at the calculated value 


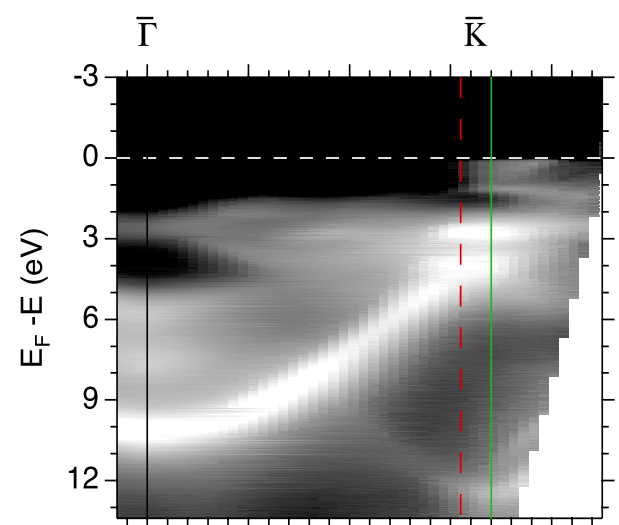

(a)

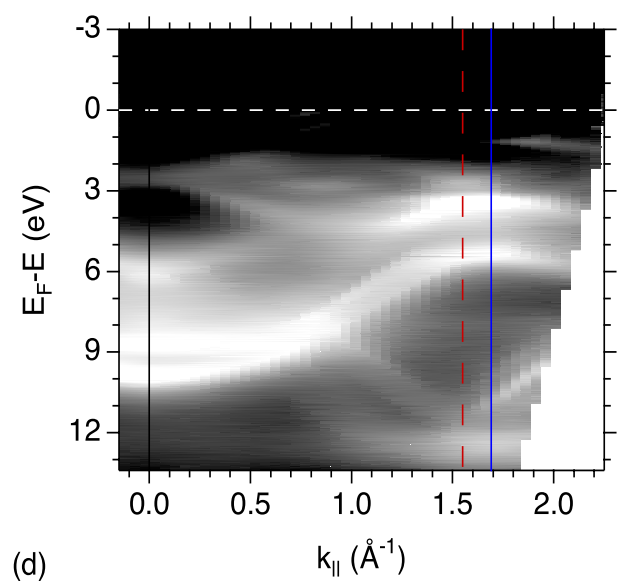

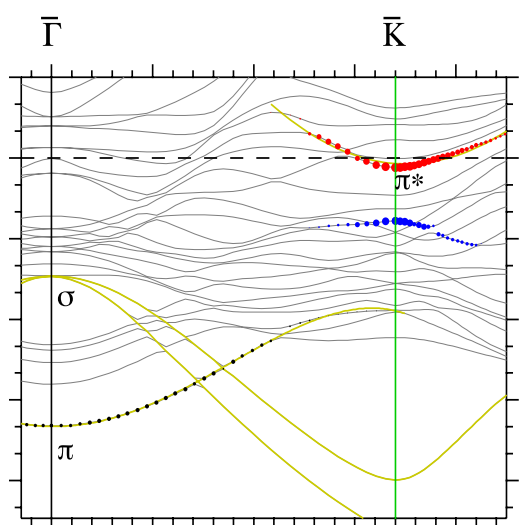

(b)



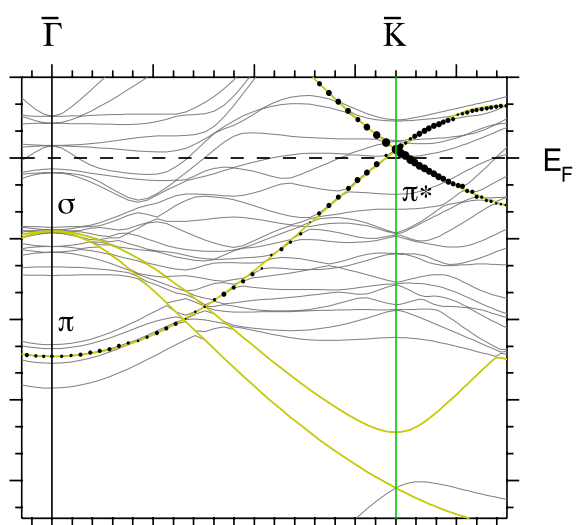

(c)

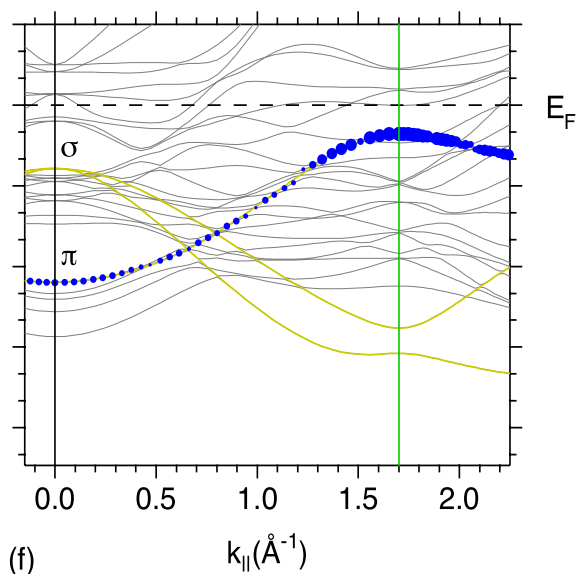

FIG. 2. (Color online) Band structures of graphene and $h$-BN nanomesh on $\mathrm{Ru}(0001)$ along $\bar{\Gamma} \overline{\mathrm{K}}$. (a) $\mathrm{He} \mathrm{II}_{\alpha}$ photoemission of $g / \mathrm{Ru}(0001)$. $[(\mathrm{b})$ and $(\mathrm{c})]$ DFT of $g / \mathrm{Ru}(0001)$ for the low $\left[\left(\mathrm{C}_{\mathrm{A}}, \mathrm{C}_{\mathrm{B}}\right) \sim(\right.$ top, hcp $\left.)\right]$ and high $\left[\left(\mathrm{C}_{\mathrm{A}}, \mathrm{C}_{\mathrm{B}}\right) \sim(\mathrm{hcp}\right.$, fcc $\left.)\right]$ regions, respectively. (d) He II $\alpha$ photoemission of $h$-BN/Ru(0001). [(e) and (f)] DFT of $h$-BN/Ru(0001) for the low $[(\mathrm{B}, \mathrm{N}) \sim(\mathrm{fcc}$, top $)]$ and high $[(\mathrm{B}, \mathrm{N}) \sim(\mathrm{hcp}, \mathrm{fcc})]$ regions, respectively. The vertical lines at $\overline{\mathrm{K}}$ indicate the boundaries of the $1 \times 1$ surface Brillouin zones for Ru (red dashed), $h$-BN (blue solid), and graphene (green solid). The size of the filled circles in (b), (c), (e), and (f) represents the $p_{z}$ weight of the adsorbate atoms on the bands, where blue describes $\mathrm{C}_{\mathrm{A}}$ (top) in (b), and $\mathrm{N}$ in (e) (top) and (f) (fcc). Red describes hollow site atoms [ $\mathrm{C}_{\mathrm{B}}$ in (b) and B in (e)]. Black circles depict the average of the two inequivalent adsorbate atoms. Thick yellow curves are guides for the eyes.

for free-standing graphene, a four-layer $\mathrm{Ru}$ slab with the lattice constant adjusted, a $36 \times 36 \times 1 k$ sampling, and $400 \mathrm{eV}$ cutoff. ${ }^{28}$ The electrostatic potential calculation has been performed with one $g$ layer on both sides of a five-layer Ru slab with the same lateral periodicity as for the structural calculations and the vertical positions as they result from the latter. The whole supercell had a vertical height of $50 \AA$ leading to an effective vacuum region larger than $30 \AA$.

\section{RESULTS AND DISCUSSION}

The hybridization of the carbon $p_{z}$ orbitals with the substrate atoms breaks the symmetry between the $C_{A}$ and the $C_{B}$ atoms. This is reflected in the band structure where a large $\pi$ band gap opens at $\overline{\mathrm{K}}$. Figure 2(a) shows the measured band structure for $g / \mathrm{Ru}(0001)$ along $\bar{\Gamma} \overline{\mathrm{K}}$. At $\overline{\mathrm{K}}$ the $\pi$ band levels off at a binding energy of $4.6 \pm 0.1 \mathrm{eV}$. The strong hybridization is in line with observations on $g / \mathrm{Ni}(111) .{ }^{29}$ The experiment is in good agreement with calculations for a $1 \times 1$ graphene sheet $2.2 \AA$ above the topmost $\mathrm{Ru}$ layer with
$\left(\mathrm{C}_{\mathrm{A}}, \mathrm{C}_{\mathrm{B}}\right) \sim($ top, hcp) $\quad$ [Fig. 2(b)]. Calculations with $\left(\mathrm{C}_{\mathrm{A}}, \mathrm{C}_{\mathrm{B}}\right) \sim(\mathrm{hcp}, \mathrm{fcc}) 3.7 \AA$ above the $\mathrm{Ru}$ top layer result in a shift of the carbon derived bands to lower binding energies. The $\pi$ and $\pi^{*}$ bands get connected with Dirac cones at $\mathrm{K}$, as it is expected for freestanding graphene ${ }^{30}$ [Fig. 2(c)]. The experiment does not, however, indicate a $\pi$ band splitting, which is two different $\pi$ bands for high and low graphene. With a recent paper on a $\mathrm{C} 1 s$ core-level splitting as measured with X-ray photoelectron spectroscopy (XPS) in $g / \mathrm{Ru}(0001)$ (Ref. 16) that was assigned to the high and low regions, this suggests that the high regions are electronically linked to the low regions in a way that hampers the free graphene picture as it emerges in Fig. 2(c). The same section of $k$-space for $h$-BN/Ru(0001) is shown in Fig. 2(d). The $\pi$ band levels off at a binding energy of $5.4 \pm 0.1 \mathrm{eV}$. Calculations for a $1 \times 1(\mathrm{~B}, \mathrm{~N}) \sim($ fcc, top $)$ sheet $2.2 \AA$ above the topmost Ru layer describe the global characteristics of the $\sigma$ bands and the $\pi$ band of $h$-BN on Ru well [Fig. 2(e)]. For the case of the $h$-BN nanomesh, a $\pi$ band splitting is observed in experiment and theory [Figs. 2(d)-2(f)], which is also consistent with the N $1 s$ core-level splitting. ${ }^{31}$ This signifies an 


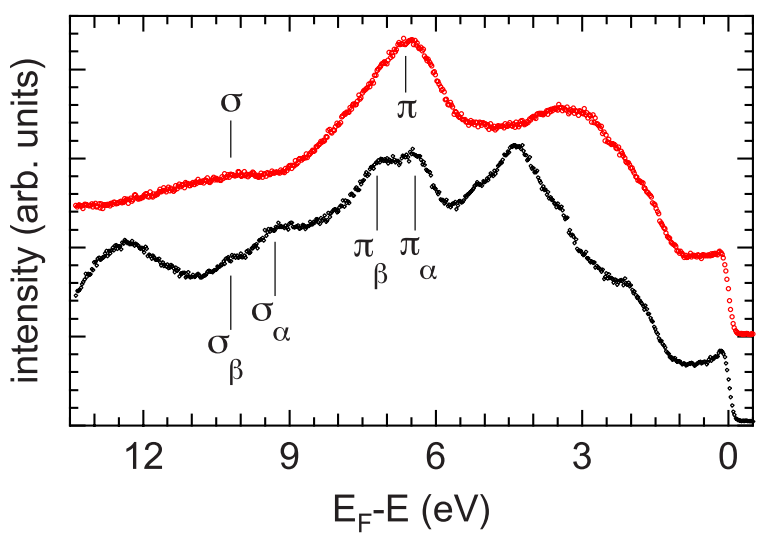

FIG. 3. (Color online) $\mathrm{He} \mathrm{II}_{\alpha}$ energy distribution curves at $k_{\|}$ $=1.12 \AA^{-1}$ for graphene (red open circles) and $h$-BN nanomesh (black open diamonds) on $\mathrm{Ru}(0001)$ extracted from the band structure data of Figs. 2(a) and 2(d), respectively. The curves are vertically offset for clarity.

intriguing but important difference between graphene and the $h$-BN nanomesh on $\mathrm{Ru}(0001)$, and is proposed to lie in the difference in the atomic and the electronic structure (see below). Theory additionally shows that the two atoms in the base of the $s p^{2}$ networks have a different $p_{z}$ weight on the different bands. For the case of $h$-BN in the low region (holes), the $\pi$ band is mainly nitrogen derived while the unoccupied $\pi^{*}$ band has its main weight on the boron atoms. For graphene in the low region (valley) only the $\mathrm{C}_{\mathrm{B}}$ atom contributes to the $\pi^{*}$ band close to $\overline{\mathrm{K}}$ while the $\pi$ band has equal portions from top and hollow site atoms. The effect of $p_{z}-d$, that is $\mathrm{C}(\mathrm{N})-\mathrm{Ru}$ hybridization, is reflected in the low regions (valley and holes) by states in the $\pi$ band gap that are mainly $\mathrm{C}_{\mathrm{A}}(\mathrm{N})$-Ru derived. In the case of the high regions of graphene (mounds), the two carbon atoms are degenerate again, and consequently $\mathrm{C}_{\mathrm{A}}$ and $\mathrm{C}_{\mathrm{B}}$ contribute in equal measures to the $\pi$ band. For the weakly bound BN (wires) the nitrogen and the boron atoms determine the $\pi$ and the $\pi^{*}$ band, respectively.

A distinct difference between graphene and the $h$-BN nanomesh is the band splitting of $0.8 \mathrm{eV}$ for the $\mathrm{BN}$ bands that belong to the "wires" $\left(\sigma_{\alpha}\right.$ and $\left.\pi_{\alpha}\right)$ and the "holes" $\left(\sigma_{\beta}\right.$ and $\pi_{\beta}$ ), respectively [Figs. 2(d) and 3]. The band splitting of $h$-BN was assigned to the dielectric nature of $h$ - $\mathrm{BN}$ and the local work function difference between the hole and the wire regions. ${ }^{6}$ For graphene no splitting is observed, where a splitting smaller than $310 \mathrm{meV}$ could not be resolved by the experiment [Figs. 2(a) and 3]. This can be explained by a smaller corrugation of the graphene layer or by the metallic nature of graphene. Although both systems are corrugated isoelectric $s p^{2}$ networks where atoms reside in two different vertical positions (low and high regions) which leads to distinguishable core-level binding energies, this turns out to be not sufficient to conclude on an equivalent valence band structure of $g / \mathrm{Ru}$ and $h$-BN/Ru.

The metallicity of $g / \operatorname{Ru}(0001)$ is reflected in the measured Fermi surface map (FSM) which is compared to that of $h$-BN/Ru(0001) in Fig. 4. The FSM of $h-\mathrm{BN} / \mathrm{Ru}(0001)$ shows only bands that are also seen on the bare $\mathrm{Ru}(0001)$
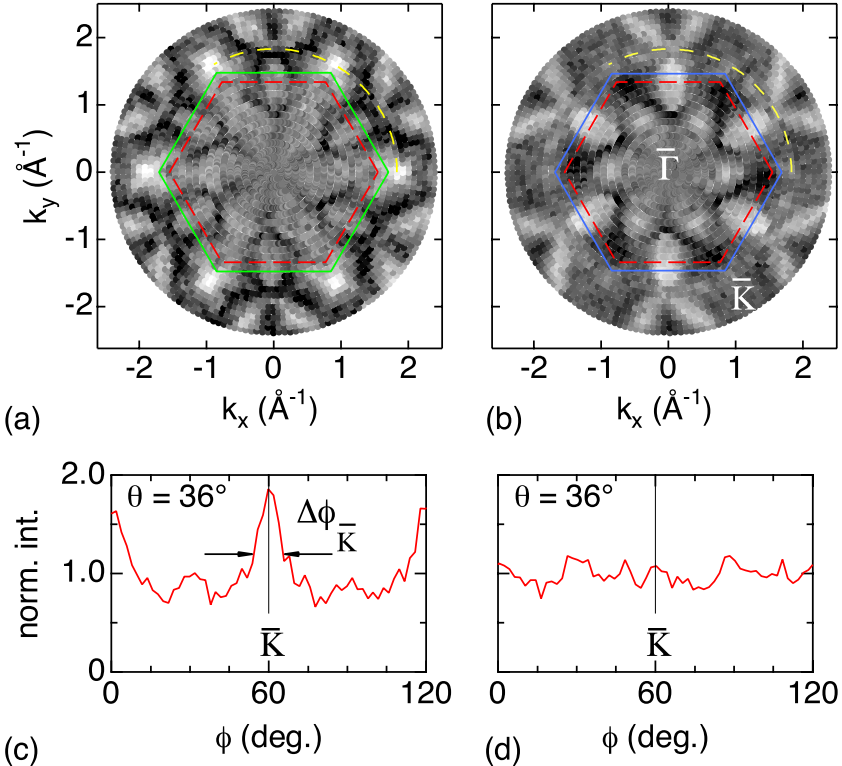

FIG. 4. (Color online) He II $\alpha$ Fermi surface maps. (a) $g / \mathrm{Ru}(0001)$. (b) $h-\mathrm{BN} / \mathrm{Ru}(0001)$. The hexagons indicate the surface Brillouin zones of $\mathrm{Ru}(0001)$ (red dashed), graphite [green solid in (a)], and $h$-BN [blue solid in (b)]. (c) and (d) show the normalized intensities of azimuthal cuts along the dashed yellow sectors in (a) and (b), respectively.

surface (data not shown). On the other hand, $g / \operatorname{Ru}(0001)$ displays states at the Fermi level that are reminiscent to the Dirac points at the $\overline{\mathrm{K}}$ points of free-standing graphene. ${ }^{30}$ The band structure measurements in Fig. 2(a) demonstrate that these graphene related states are part of the $\pi^{*}$ band, which means that charge is transferred from the substrate to the graphene. The Luttinger volume of the electron pockets near $\mathrm{K}$ corresponds to the number of transferred electrons $N_{e}$ $=2 \pi /(3 \sqrt{3})\left(\Delta \phi_{\overline{\mathrm{K}}}\right)^{2}$, where $\Delta \phi_{\overline{\mathrm{K}}}$ is taken as the full width at half maximum of the intensity on an azimuthal cut across $\overline{\mathrm{K}}$, in radians [Fig. 4(c)]. For the FSM in Fig. 4(a) we find $\Delta \phi_{\overline{\mathrm{K}}}=0.27 \pm 0.03 \mathrm{rad}$, which translates to $N_{e}=0.09 \pm 0.02$ electrons per $1 \times 1$ graphene unit cell. Of course, this value is the weighted average for mounds and valleys.

The differences in the atomic and electronic structure are also reflected in the potential energy surfaces that drive the functionality of the superstructures as templates for the formation of molecular arrays. In Fig. 5(a) the calculated electrostatic potential for $g / \mathrm{Ru}(0001)$ at the position of adsorbed xenon atom cores $(3.8 \AA$ above the cores of the outermost carbon atoms) is shown. As for the case of the $h$-BN/ $\mathrm{Rh}(111)$ nanomesh, the electrostatic energy landscape correlates with the atomic corrugation. ${ }^{8}$ For the theoretical corrugation of $g / \mathrm{Ru}(0001)$ of $1.5 \AA$, we obtain a potential amplitude of $0.52 \mathrm{eV}$. This potential amplitude may be measured with photoemission of adsorbed xenon (PAX). ${ }^{32}$ Also for $g / \mathrm{Ru}(0001)$ two Xe bonding regions can be distinguished with distinct $\mathrm{Xe} 5 p_{1 / 2}$ photoemission binding energies [Fig. 5(b)]. Assuming a Xe van der Waals radius of $2.2 \AA, 50 \mathrm{Xe}$ atoms per $g / \mathrm{Ru}(0001)$ unit cell are expected for the monolayer coverage. ${ }^{17}$ From the spectral weight and the known atomic structure, it can be deduced that the $\mathrm{Xe}^{V}$ species in 
(a)

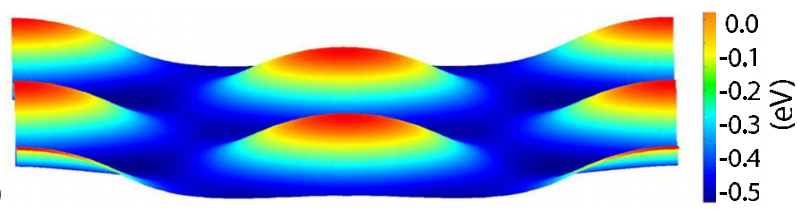

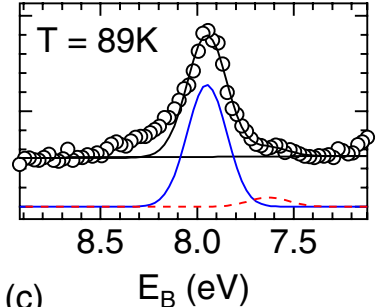

(c)

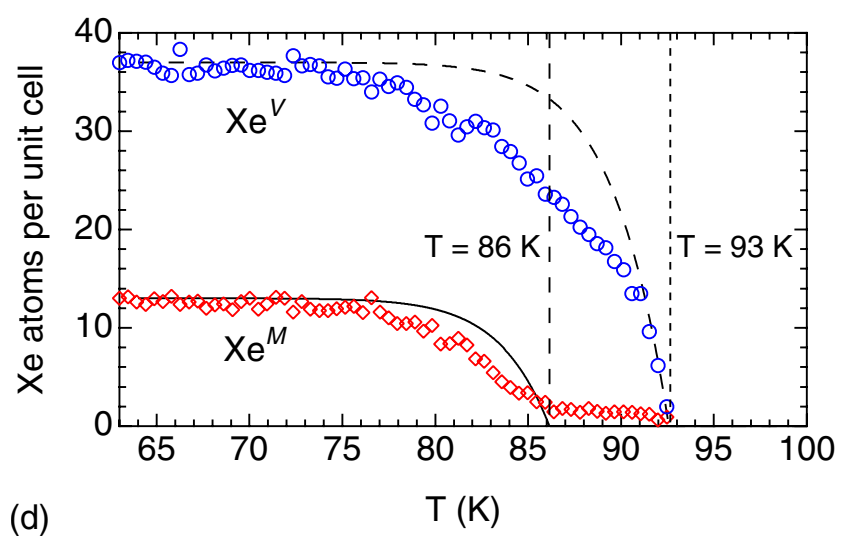

FIG. 5. (Color online) Xe adsorption on $g / \mathrm{Ru}(0001)$. (a) Calculated electrostatic potential map $3.8 \AA$ above the outermost carbon atoms. [(b) and (c)] He $\mathrm{I}_{\alpha}$ excited Xe $5 p_{1 / 2}$ spectra for two Xe coverages during thermal desorption. At high coverage two spectral components may be distinguished, as can be seen from the two Gaussians that are fitted to the data. (d) Spectral weights of the two $\mathrm{Xe}$ species as a function of temperature. Blue open circles stand for the high binding energy and red open diamonds for the low binding energy component.

the graphene valley has the higher $\mathrm{Xe} 5 p_{1 / 2}$ photoemission binding energy and contains 37 atoms at full coverage. The $\mathrm{Xe}^{M}$ species with lower photoemission binding energy corresponds to Xe adsorbed on the mounds of the graphene. The binding energy difference between these two species of $236 \pm 5 \mathrm{meV}$ is determined from a fit of two Gaussians with equal width and does not depend on the coverage. This value reflects a local work function difference between $V$ and $M$ graphene and is in line with the theoretical result shown in Fig. 5(a). Since for $h$-BN/Ru(0001) no experimental PAX data are available we compare the $g / \mathrm{Ru}(0001)$ PAX results with the similar system $h$-BN/Rh(111) nanomesh. ${ }^{8}$ For both, the strongly bound regions (valley and holes) have a lower work function. However, for $g / \mathrm{Ru}(0001)$ the local work function difference is about $20 \%$ lower than for the case of $h$-BN/ Rh(111). Intuitively, this is related to the metallic nature of the graphene that screens out lateral electric fields in the $s p^{2}$ layer. The better screening of graphene is indeed reflected in the $\mathrm{Xe} 5 p_{1 / 2}$ final-state binding energy as referred to the vacuum level $E_{B}^{V}=E_{B}+\Phi .{ }^{33}$ The values in Table I show
TABLE I. Experimentally determined Xe parameters for $g / \mathrm{Ru}(0001)$ and $h$-BN/Rh(111) (Ref. 8): desorption energies $E_{d}$ and number of Xe atoms at full coverage $N_{1}$. For all fits an attempt frequency $\nu$ of $1.2 \times 10^{12} \mathrm{~Hz}$ has been used. Xe $5 p_{1 / 2}$ photoemission binding energies $E_{B}$ and global work functions $\Phi$ for the two coverage limits $(\theta \rightarrow 0=$ zero coverage, $\theta \rightarrow 1=$ monolayer coverage). The errors for the binding energies $E_{B}$ and the work functions $\Phi$ are $\pm 0.02 \mathrm{eV}$ and $\pm 2 \mathrm{meV}$ for the desorption energies $E_{d}$.

\begin{tabular}{lccccc}
\hline \hline & \multicolumn{2}{c}{$g / \mathrm{Ru}(0001)$} & \multicolumn{3}{c}{$h$-BN/Rh(111) } \\
Phase & $\mathrm{Xe}^{M}$ & $\mathrm{Xe}^{V}$ & $C^{W}$ & $C^{H}$ & $R^{H}$ \\
\hline$E_{d}(\mathrm{meV})$ & 222 & 231 & 181 & 184 & 208 \\
$N_{1}$ & 13 & 37 & 25 & 17 & 12 \\
$E_{B}(\theta \rightarrow 0)(\mathrm{eV})$ & & 7.97 & & & 7.87 \\
$E_{B}(\theta \rightarrow 1)(\mathrm{eV})$ & 7.56 & 7.80 & 7.42 & & 7.72 \\
$\Phi(\theta \rightarrow 0)(\mathrm{eV})$ & & 3.90 & & & 4.18 \\
$\Phi(\theta \rightarrow 1)(\mathrm{eV})$ & & 3.89 & & & 4.18 \\
\hline \hline
\end{tabular}

that $E_{B}^{V}$ of $\mathrm{Xe}^{V}$ at monolayer coverage $(\theta \rightarrow 1)$ on $g / \mathrm{Ru}(0001)$ is $210 \mathrm{meV}$ smaller than that of $\mathrm{Xe}^{H}$ on $h$-BN/Rh(111).

From the thermal desorption the Xe adsorption energy is inferred. In Fig. 5(d) the spectral weights of the Xe species are shown as a function of temperature (heating rate $\beta$ $=1.5 \pm 0.05 \mathrm{~K} / \mathrm{min}$ ). The temperatures at which the two Xe species disappear indicate that $\mathrm{Xe}^{V}$ is up to about $8 \%$ stronger bound than $\mathrm{Xe}^{M}$. In order to compare $g / \mathrm{Ru}$ with $h$-BN/Rh, the temperature-dependent weights of the two Xe species were fitted to zero-order desorption. From $-d N$ $=\nu / \beta \exp \left(-E_{d} / k_{B} T\right) d T$ the desorption energies $E_{d}$ are found (see Table I). The values are slightly smaller than the desorption energies on graphite [249 meV (Ref. 34)] but higher with respect to $h$-BN/Rh. The fits for zero-order desorption show that the $\mathrm{Xe}^{V}$ species are not well described with a single desorption energy [dashed line in Fig. 5(d)]. In the $h$-BN/Rh case two $\mathrm{Xe}^{H}$ phases $\left(C^{H}\right.$ and $\left.R^{H}\right)$ had been identified by a pronounced kink in the desorption spectrum of $\mathrm{Xe}^{H}$. The more strongly bound of these two phases was assigned to $\mathrm{Xe}^{H}$ atoms at the rims of the holes where dipole rings induce an enhanced polarization and bonding. For $g / \mathrm{Ru}(0001)$ a clear kink is not visible but the deviations from the fit also indicate variations in bonding strength of the $\mathrm{Xe}^{V}$ atoms. It could well be the case that the stronger $\mathrm{Xe}$ bonding sites are localized at the rims of the $g / \mathrm{Ru}$ valley. The difference to $h$-BN/Rh may be understood by the inverse shape of the potential energy surface. If we fit, like for $h$-BN/Rh(111) the $C^{H}$ and the $R^{H}$ phase, ${ }^{8}$ two $\mathrm{Xe}^{V}$ phases for $g / \mathrm{Ru}(0001)$, binding energies of 222 and $234 \mathrm{meV}$ are obtained for $g / \mathrm{Ru}(0001)$. The smaller difference of $12 \mathrm{meV}$ is not unreasonable if the lower local work function modulation of $g / \mathrm{Ru}$ is considered, which induces a lower polarization of $\mathrm{Xe}$ on the rims.

\section{CONCLUSIONS}

In conclusion, both $g / \mathrm{Ru}$ and $h$-BN/Ru have strongly bound and weakly bound regions but with inverted topogra- 
phy. The presented findings suggest that $g / \mathrm{Ru}$ is not a corrugated single-layer dielectric like $h$-BN/Ru but a corrugated single-layer metal with a Fermi surface. In contrast to $h$-BN/Ru, $g / \mathrm{Ru}$ shows no $\sigma$ band splitting due to a different polarization of the unequal regions in the supercell. However, also on $g / \mathrm{Ru}$ lateral electric fields are found to act on adsorbates. The electrostatic potential variations within the supercell make both templates for molecular trapping, where the metallicity of graphene imposes a stronger electronic coupling of adsorbates to the underlying substrate.

\section{ACKNOWLEDGMENTS}

Fruitful discussions with Peter Blaha and technical support by Martin Klöckner are gratefully acknowledged. We thank the Swiss National Science Foundation and the German Research Foundation for their financial support. M.L.B. thanks the Humboldt Foundation. B.W. acknowledges financial support by the Bayerisch-Französisches Hochschulzentrum. Part of this work was performed at the Swiss Light Source. *greber@physik.uzh.ch

${ }^{1}$ K. S. Novoselov, A. K. Geim, S. V. Morozov, D. Jiang, Y. Zhang, S. V. Dubonos, I. V. Grigorieva, and A. A. Firsov, Science 306, 666 (2004).

${ }^{2}$ M. Corso, W. Auwärter, M. Muntwiler, A. Tamai, T. Greber, and J. Osterwalder, Science 303, 217 (2004).

${ }^{3}$ S. Marchini, S. Günther, and J. Wintterlin, Phys. Rev. B 76, 075429 (2007).

${ }^{4}$ Y. Pan, D. X. Shi, and H. J. Gao, Chin. Phys. 16, 3151 (2007).

${ }^{5}$ A. L. Vázquez de Parga, F. Calleja, B. Borca, M. C. G. Passeggi, Jr., J. J. Hinarejos, F. Guinea, and R. Miranda, Phys. Rev. Lett. 100, 056807 (2008).

${ }^{6}$ A. Goriachko, Y. B. He, M. Knapp, H. Over, M. Corso, T. Brugger, S. Berner, J. Osterwalder, and T. Greber, Langmuir 23, 2928 (2007).

${ }^{7}$ S. Berner et al., Angew. Chem., Int. Ed. 46, 5115 (2007).

${ }^{8}$ H. Dil, J. Lobo-Checa, R. Laskowski, P. Blaha, S. Berner, J. Osterwalder, and T. Greber, Science 319, 1824 (2008).

${ }^{9}$ The lattice mismatch $\mathfrak{M}$ is defined as the relative difference $\mid a_{s}$ $-a_{s p^{2}} \mid / a_{s}$ between the substrate in-plane lattice constant $a_{s}$ and the $s p^{2}$ network lattice constant $a_{s p^{2}}$.

${ }^{10}$ M. T. Paffett, R. J. Simonson, P. Papin, and R. T. Paine, Surf. Sci. 232, 286 (1990).

${ }^{11}$ Y. Gamou, M. Terai, A. Nagashima, and C. Oshima, Sci. Rep. Res. Inst. Tohoku Univ. A 44, 211 (1997).

${ }^{12}$ M. Morscher, M. Corso, T. Greber, and J. Osterwalder, Surf. Sci. 600, 3280 (2006).

${ }^{13}$ T. A. Land, T. Michely, R. J. Behm, J. C. Hemminger, and G. Comsa, Surf. Sci. 264, 261 (1992).

${ }^{14}$ Y. Gamo, A. Nagashima, M. Wakabayashi, M. Terai, and C. Oshima, Surf. Sci. 374, 61 (1997).

${ }^{15}$ A. T. N'Diaye, S. Bleikamp, P. J. Feibelman, and T. Michely, Phys. Rev. Lett. 97, 215501 (2006).

${ }^{16}$ A. B. Preobrajenski, M. L. Ng, A. S. Vinogradov, and N. Mårtensson, Phys. Rev. B 78, 073401 (2008).

${ }^{17}$ D. Martoccia et al., Phys. Rev. Lett. 101, 126102 (2008).

${ }^{18}$ O. Bunk, M. Corso, D. Martoccia, R. Herger, P. R. Willmott, B. D. Patterson, J. Osterwalder, I. van der Veen, and T. Greber, Surf. Sci. 601, L7 (2007).

${ }^{19}$ B. Wang, M. L. Bocquet, S. Marchini, S. Günther, and J.
Wintterlin, Phys. Chem. Chem. Phys. 10, 3530 (2008).

${ }^{20}$ R. Laskowski and P. Blaha, J. Phys.: Condens. Matter 20, 064207 (2008).

${ }^{21}$ R. Laskowski, P. Blaha, T. Gallauner, and K. Schwarz, Phys. Rev. Lett. 98, 106802 (2007).

${ }^{22}$ B. Wang, M.-L. Bocquet, S. Günther, and J. Wintterlin, Phys. Rev. Lett. 101, 099703 (2008).

${ }^{23}$ A. L. Vázquez de Parga, F. Calleja, B. Borca, M. C. G. Passeggi, Jr., J. J. Hinarejos, F. Guinea, and R. Miranda, Phys. Rev. Lett. 101, 099704 (2008).

${ }^{24}$ T. Greber, O. Raetzo, T. J. Kreutz, P. Schwaller, W. Deichmann, E. Wetli, and J. Osterwalder, Rev. Sci. Instrum. 68, 4549 (1997).

${ }^{25}$ M. Hoesch, T. Greber, V. N. Petrov, M. Muntwiler, M. Hengsberger, W. Auwärter, and J. Osterwalder, J. Electron Spectrosc. Relat. Phenom. 124, 263 (2002).

${ }^{26}$ G. Kresse and D. Joubert, Phys. Rev. B 59, 1758 (1999).

${ }^{27}$ J. P. Perdew, K. Burke, and M. Ernzerhof, Phys. Rev. Lett. 77, 3865 (1996).

${ }^{28}$ For the calculation of the band structure of the $1 \times 1$ models, we found it more suitable to fix the graphene lattice at the relaxed value of the free-standing layer (and adjust the $\mathrm{Ru}$ substrate accordingly) because the bandwidth sensitively depends on the small strains of the graphene lattice. The Dirac point on the weakly interacting $M$ regions [Fig. 2(c)] is then slightly above $E_{\mathrm{F}}$ ( $p$ doping). In the opposite case, when the Ru lattice is kept at the relaxed value of the bare surface and the graphene is adjusted (as in Ref. 19), the Dirac point moves below $E_{\mathrm{F}}(n$ doping).

${ }^{29}$ A. Nagashima, N. Tejima, Y. Gamou, T. Kawai, and C. Oshima, Phys. Rev. B 51, 4606 (1995).

${ }^{30}$ A. Bostwick, T. Ohta, T. Seyller, K. Horn, and E. Rotenberg, Nat. Phys. 3, 36 (2007).

${ }^{31}$ A. B. Preobrajenski, M. A. Nesterov, M. L. Ng, A. S. Vinogradov, and N. Mårtensson, Chem. Phys. Lett. 446, 119 (2007).

${ }^{32}$ K. Wandelt, J. Vac. Sci. Technol. A 2, 802 (1984).

${ }^{33}$ G. Kaindl, T. C. Chiang, D. E. Eastman, and F. J. Himpsel, Phys. Rev. Lett. 45, 1808 (1980)

${ }^{34}$ H. Ulbricht, R. Zacharia, N. Cindir, and T. Hertel, Carbon 44, 2931 (2006). 